\title{
Why we should be worried about community care: a personal view of the Eighth Annual TAPS Conference*
}

\author{
Paul Crichton, Senior Registrar, The Maudsley Hospital, Denmark Hill, \\ London SE5 8AZ
}

In 1985 , nearly two years after the announcement of the intended closure of Claybury and Friern Hospitals, the Team for the Assessment Psychiatric Services (TAPS) was set up to evaluate this policy. TAPS has now grown to a staff of six full-time researchers (three psychologists, a psychiatrist, a sociologist and a health economist) and is funded mainly by the North East Thames Regional Health Authority, but also by the Department of Health. This was the eighth annual conference, and the first since the closure of Friern Hospital in March 1993.

The morning sessions gave an overview of the most recent follow-up studies on three groups of patients discharged from hospital. In summary, the long-stay, mainly schizophrenic, patients seem to be doing well, the psychogeriatric patients with "functional" (nonorganic) illness appear to be doing less well and the acute patients give cause for alarm.

The five-year follow-up of the long-stay patients presented by Professor Leff showed that these patients had fewer behavioural abnormalities, experienced less anxiety and social unease, appreciated their freedom more and had more and better social contacts (as gauged by the number of confidants and contacts with ordinary members of the public), compared with the baseline measures on the same patients in hospital. Professor Leff noted that it would have been methodologically preferable to compare these patients with matched controls still in hospital, but this would have posed practical and ethical problems. He also warned against an overoptimistic interpretation of these findings, as these patients were among the first to be discharged and were probably less severely disturbed than those discharged later ("creaming-off" effect).

Professor Knapp, a health economist, estimated the average weekly costs for these patients at $£ 519$ per person, $80 \%$ of which was spent on accommodation. Male sex, not having a partner, longer stay and higher needs in hospital (but not psychiatric diagnosis) were associated with higher cost. Although it is thought that the overall expenditure on discharged patients is somewhat lower than the average hospital cost, Professor Knapp pointed out

*Held on 15 July 1993 at the Royal Free Hospital School of Medicine, Rowland Hill Street, London NW3. that increased cost seems to be associated with better outcome. Health politicians appear to have taken note of the former, but to have ignored the latter.

In a comparison between 36 psychogeriatric patients with "functional" illness and 42 similar patients in the community (presented by $\operatorname{Dr} \mathbf{N}$. Triemann) the patients in the community showed no behavioural, physical or psychiatric deterioration and less cognitive impairment. However there seemed to be some diagnostic confusion in this study: although patients with organic dementia had initially been excluded from the study, many of the patients in the study now have Mini-Mental State scores well within the dementia range. Have they developed organic dementia, confusional states or depressive "pseudodementia" in addition to their "functional" illness?

From the Acute Services Study Dr R. Sammut reported a $\mathbf{4 0 \%}$ increase in psychotic patients thought to require admission and a $12 \%$ increase in the number of patients actually admitted, with back pressure on day hospitals, medical wards and other hospitals outside the district. There was also a $25 \%$ increase in patients admitted under section.

The four parallel afternoon sessions picked out details from this panorama and included contributions from researchers in Israel, Spain, New York and New Zealand. Among the topics discussed were views of relatives on care for the elderly, environmental assessment, in-patient community care for the elderly, the Hampstead Schizophrenia Surveys 1986-1991, care of schizophrenic patients in general practice, the clubhouse model of social and work rehabilitation, long-stay affective patients, care programming, case management, quality of life for patients in residential care, community hostels and homes, and the Cambridge Sheltered Housing Survey.

The National Schizophrenia Fellowship estimates that about 40 people have been killed by 27 schizophrenic patients in England since April 1991, i.e. during a period of accelerated bed closure. Clearly we need to know if this marks an increase compared with, say, five or ten years ago, and, if so, whether it can be seen as part of an increase in violent crime in the general population or is more closely related to 
the policy of closing psychiatric beds and hospitals. The forensic implications of community care, TAPS reports a $40 \%$ increase in the number of acute obviously of great concern to the public and media, patients thought to need admission, but only a $12 \%$ should be revealed by further exploration of this rich increase in the number who were actually admitted. source of data.

\section{The Association of Pakistani Psychiatrists}

This Organisation (APP) has been set up to provide a forum for the exchange of information and ideas and to bring together the views and experiences of colleagues working in psychiatry in the UK.

One of the immediate aims of the organisation is to set up a scientific journal for circulation in the UK and in Pakistan. Contributions will be invited from practitioners in both countries, with an emphasis on clinical and epidemiological aspects of psychiatry.

The professional and educational remit of the APP will be to examine the possibility of providing final year student elective placements, participation in the
Overseas Doctors' Training Scheme, and helping out in areas previously identified as requiring assistance, such as preparation of curriculum vitae and examination techniques. We also intend to hold regular academic meetings comprising internal contributions as well as speakers invited specifically for their expertise in the subject under discussion.

The first meeting of the Executive Committee has taken place and we are now in the process of launching a membership drive. All enquiries should be sent to Dr Ahmad Mahmood, Association of Pakistani Psychiatrists, 18 Hardy Close, Hinckley, Leicestershire, LE10 IPF (Telephone 0455 250842). 